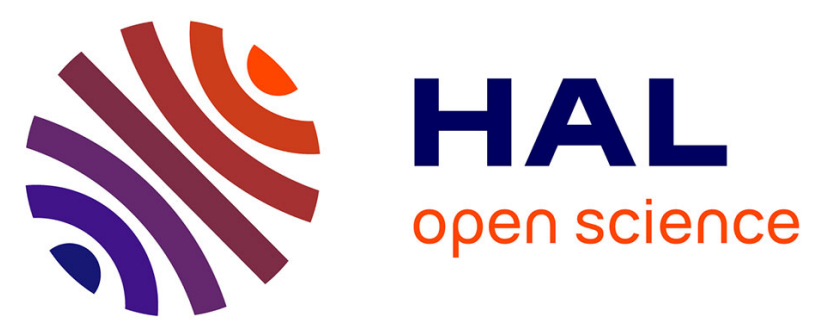

\title{
The effect of excessive fat tissue on the measure of bone mineral density by dual-X ray absorptiometry: the impact of substantial weight loss following sleeve gastrectomy
}

Laurent Maïmoun, Denis Mariano-Goulart, Audrey Jaussent, Patrick Lefebvre, Marie-Christine Picot, Krishna Mahadea, Vincent Boudousq, Clémence Fouillade, David Nocca, Fayçal Ben Bouallègue

\section{To cite this version:}

Laurent Maïmoun, Denis Mariano-Goulart, Audrey Jaussent, Patrick Lefebvre, Marie-Christine Picot, et al.. The effect of excessive fat tissue on the measure of bone mineral density by dual-X ray absorptiometry: the impact of substantial weight loss following sleeve gastrectomy. Clinical Physiology and Functional Imaging, 2019, 39 (5), pp.345-354. 10.1111/cpf.12584 hal-02131102

\author{
HAL Id: hal-02131102 \\ https://hal.science/hal-02131102
}

Submitted on 12 Jun 2020

HAL is a multi-disciplinary open access archive for the deposit and dissemination of scientific research documents, whether they are published or not. The documents may come from teaching and research institutions in France or abroad, or from public or private research centers.
L'archive ouverte pluridisciplinaire HAL, est destinée au dépôt et à la diffusion de documents scientifiques de niveau recherche, publiés ou non, émanant des établissements d'enseignement et de recherche français ou étrangers, des laboratoires publics ou privés. 


\section{The effect of excessive fat tissue on the measure of bone mineral density by dual X-ray absorptiometry: the impact of substantial weight loss following sleeve gastrectomy}

Laurent Maïmoun ${ }^{1,2}$, Denis Mariano-Goulart ${ }^{1,2}$, Audrey Jaussent ${ }^{3}$, Patrick Lefebvre ${ }^{4}$, Marie-Christine Picot ${ }^{3}$, Krishna Mahadea ${ }^{5}$, Vincent Boudousq ${ }^{6}$, Clémence Fouillade ${ }^{7}$, David Nocca ${ }^{7}$ and Fayçal Ben Bouallègue ${ }^{1,2}$

${ }^{1}$ Service de Médecine Nucléaire, Hôpital Lapeyronie, Centre Hospitalier Régional Universitaire (CHRU) Montpellier et Université Montpellier (UM), Montpellier, France, ${ }^{2}$ Physiologie et Médecine Expérimentale du Coeur et des Muscles (PhyMedExp), University of Montpellier, INSERM U1046, CNRS, UMR 9214, Montpellier Cedex 5, France, ${ }^{3}$ Unité de Recherche Clinique, Biostatistiques et Epidémiologie, Département de l'Information Médicale, CHRU de Montpellier, Montpellier, France, ${ }^{4}$ Departement d'Endocrinology, Diabetes, Nutrition, Hôpital Lapeyronie, CHRU Montpellier, Montpellier, France, ${ }^{5}$ Université de Montpellier, Montpellier, France, ${ }^{6}$ Service de Médecine Nucléaire, Hôpital Carémeau, Nîmes, France, and ${ }^{7}$ Service de Chirurgie Digestive A, Hôpital Saint Eloi, CHRU Montpellier, Montpellier, France

\section{Summary}

\section{Correspondence}

Laurent Maïmoun, Département de Biophysique, Université de Montpellier, Service de Médecine Nucléaire, Hôpital Lapeyronie, 371, avenue du Doyen Gaston Giraud, 34295 Montpellier Cedex 5, Montpellier, France.

E-mail: 1-maimoun@chu-montpellier.fr

\section{Key words}

bone mineral density; dual-energy X-ray absorptiometry; fat mass; obese; overweight; underestimation
Purpose Dual-energy X-ray absorptiometry (DXA) is used in clinical routine to determine areal bone mineral density (aBMD). However, it is not clear whether excessive fat mass or substantial weight loss modify the aBMD measurements. The aim of this study was to evaluate the effect of soft tissue composition on aBMD measured by DXA using a clinical model (i.e. sleeve gastrectomy: SG) that induces substantial body weight loss.

Methods Areal bone mineral density and body composition (fat mass: FM and lean tissue mass: LTM) were determined by DXA in 41 obese patients (33 women, $80.5 \%$ ) just before SG and 1 month later.

Results One month after SG, mean weight loss was $-9.8 \pm 2.6 \mathrm{~kg}$, with a significant decrease in LTM and FM (kg) ranging from $-7.3 \%$ to $-9.5 \%$. The relative variation in aBMD was increased at the lumbar spine $(2.45 \pm 3.44 \%)$ and decreased at the hip $(-1.47 \pm 2.28 \%)$, whereas no variation was observed for the whole body and radius. The variation in aBMD at the lumbar spine was inversely correlated with variations in weight, whole-body FM and trunk FM, but not LTM.

Conclusion This study shows evidence of a potential effect of body composition, particularly FM, on aBMD. However, given the modest change in aBMD, which was close to the precision error of aBMD measurements, it appears that significant weight loss does not have a clinically significant impact on the evaluation of aBMD using DXA.

\section{Introduction}

Dual-energy X-ray absorptiometry (DXA) is the reference technique to evaluate areal bone mineral density [areal bone mineral density (aBMD), $\left.\mathrm{g} \mathrm{cm}^{-2}\right]$, a parameter that is directly related to bone strength. The basic principle of DXA is the measurement of X-ray transmission at high and low energies throughout the body. The X-ray beam energies are attenuated during the passage through tissues, and this attenuation is influenced by the intensity of the effective photon energy and thickness, as well as the tissue composition [adipose tissue, lean tissue (muscle) and bone] (Bazzocchi et al., 2016).
Consequently, accurate aBMD measurement may be influenced by soft tissue, particularly in clinical situations of patients presenting large amounts of adipose tissue, such as obesity. Previous studies demonstrated that fat tissue in humans (Svendsen et al., 1993, 1995, 2002; Binkley et al., 2003) and animals (Picaud et al., 1999) modified DXA measurements and affected the accuracy of BMD measurements. Similarly, experimental studies demonstrated that BMD measurements were modified when different thicknesses of exogenous fat (lard packets) or water were positioned at various regions of the body (Evans et al., 2006; Kim et al., 2012), this effect being particularly marked at the lumbar spine (Kim et al., 2012). However, 
these strategies were artificial, and it is possible that in physiological conditions body fat and the lean component interfere differently with X-rays. In clinical conditions, obese patients with a mean weight loss of $11.3 \mathrm{~kg}$ after intervention showed a false $1 \%-2 \%$ decrease in BMD at the anterior-posterior lumbar spine as evaluated by first-generation DXA (Svendsen et al., 1993, 1995, 2002). This modest change, which is similar to the age-related aBMD reduction in postmenopausal women (Hansen et al., 1995), was attributed to a small variation in trunk soft tissue heterogeneity. However, it cannot be excluded that, over a long period of 1 year, aBMD is reduced due to high weight loss and caloric restriction (Bredella et al., 2011, 2013, 2017; Soltani et al., 2016).

In clinical settings, although obese patients generally present higher aBMD than normal-weight controls (Binkley et al., 2003; Maimoun et al., 2016, 2017), the effect of a high amount of soft tissue on DXA aBMD measurements is not precisely known. An interesting 'clinical model' to investigate this potential effect may be bariatric surgery (BS), as it induces acute and massive body weight and soft tissue [lean tissue mass (LTM) and fat mass (FM)] losses within a limited period (Maimoun et al., 2016) of 1 month, which theoretically does not allow the same magnitude of variation in aBMD.

Taking into account this background, we assumed that substantial weight loss would influence aBMD measurements. Therefore, the aim of this study was to evaluate the effects of the change in soft tissue composition induced by SG on aBMD measured by DXA. We also sought to determine whether the aBMD changes would vary with sex and anatomical site (i.e. femur, lumbar spine and radius).

\section{Subjects and Methods}

A full description of the recruitment method was reported in detail elsewhere (Maimoun et al., 2017). Briefly, study approval was obtained from the Regional Research Ethics Committee and permission for the clinical trials was granted by the French Health Products Safety Agency. Written informed consent was obtained from all participants. The clinical trial number is NCT02310178.

\section{Subjects}

Forty-one patients (33 women, 80.5\%) from 18.0 to 68.2 years old were recruited from a waiting list of candidates for obesity surgery (SG) in the Department of Surgery A at Montpellier University Hospital (France). Patients were selected for surgery if other weight-loss treatments had failed and BMI was greater than $40 \mathrm{~kg} \mathrm{~m}^{-2}$ (severe obesity) or greater than or equal to $35 \mathrm{~kg} \mathrm{~m}^{-2}$ with the presence of obesity-related comorbidities (e.g. diabetes mellitus type 2, high blood pressure or sleep apnoea syndrome) (Jensen et al., 2013).

Patients with a prosthesis or a body weight $>190 \mathrm{~kg}$ (i.e. exceeding the table's weight limit) were excluded from the study due to the limitations of the densitometry device. Moreover, patients were excluded if they were being treated for osteoporosis or had recently undergone an exam requiring injection of a contrast medium or radioisotopes (detrimental for accuracy).

\section{Methods}

All patients were evaluated the day before the operation (presurgery) and 1 month later (mean $1.13 \pm 0.35$ month) in the morning (8.30-9.00 a.m.) after an overnight fast. For each visit, standing height was measured with a stadiometer to the nearest $0.1 \mathrm{~cm}$. Weight was determined using a weight scale with a precision of $0.1 \mathrm{~kg}$. BMI was calculated as weight $(\mathrm{kg})$ divided by the square of height $(\mathrm{m})$.

\section{Areal BMD, body fat and lean tissue mass evaluation}

Bone mineral content (BMC, $\mathrm{g})$, aBMD $\left(\mathrm{g} \mathrm{cm}^{-2}\right)$ and soft tissue body composition (FM, kg; percentage of FM: \%FM; and LTM, kg) were measured by DXA at the whole body, total hip, lumbar spine and radius (Hologic QDR-4500A, Hologic, Inc., Waltham, MA, USA). Identical and accurate positioning of the region of interest (ROI) was ensured by superimposing the image from the first session (presurgery) on the image from the second session ( 1 month after SG); this initial image thus served as the visual reference. Moreover, all scanning and analyses were performed by the same operator to ensure consistency. The quality control procedure for DXA was checked daily by scanning a lumbar spine phantom consisting of calcium hydroxyapatite embedded in a cube of thermoplastic resin (DPA/QDR-1; Hologic x-calibre anthropometrical spine phantom). The CVs given by the manufacturer were $<1 \%$ for aBMD, LTM and FM. In line with recommendations from the International Society for Clinical Densitometry (ISCD), the least significant change (LSC) was calculated for 30 subjects $\left(0.013 \mathrm{~g} \mathrm{~cm}^{-2}\right.$ at lumbar spine and $0.018 \mathrm{~g} \mathrm{~cm}^{-2}$ at total hip).

\section{Statistical analysis}

Categorical parameters are expressed as numbers and percentages, and quantitative parameters are expressed as means with standard deviation (SD) and range. Shapiro-Wilk's test was used to test the normality of the continuous parameters.

Chi-square or Fisher's exact tests compared the categorical parameters between two groups, and Mann-Whitney or Student's t-tests compared the continuous parameters, depending on the normality of the distributions.

The absolute change in body composition parameters at 1 month, defined as [1-month value-presurgery value], and the percentage $(\%)$ of relative change at 1 month, defined as [100* (1 month-presurgery)/presurgery], were calculated at each site and compared between the sexes. The within-group variations were also tested for each sex and at each site. 
The relationships between the absolute changes in soft tissue parameters and the absolute change in aBMD at each site were assessed using Pearson's or Spearman's correlation coefficients, depending on the normality of the distributions. To determine the factors independently associated with the absolute variation in $\mathrm{aBMD}$ at the lumbar spine, parameters significantly associated with this absolute variation in the univariate analysis $(\mathrm{P}<0.20)$ and not collinear each other were entered into a multivariate analysis using a linear regression model.

Significance was set at $\mathrm{P}<0.05$. All analyses were performed with SAS Enterprise Guide, version 4.3 (SAS Institute, Cary, NC, USA). The graphs on Fig. 1 were generated with $\mathrm{R}$ statistical software (www.r-project.org, version 3.5.2).

\section{Results}

This study included 41 obese patients (33 women, 80.5\%) with a mean age of $39.2 \pm 14.1$ years; the main characteristics are presented in Table 1. At baseline, the male and female patients were comparable in terms of age, BMI, waist circumference and whole body, trunk and upper limb FM, but the men presented significantly greater weight, height and LTM at all sites (whole body, upper limbs, lower limbs) and lower limb FM.

After 1 month, the absolute mean weight loss was $-9.8 \pm 2.6 \mathrm{~kg}$, but the men presented a significantly higher absolute $(-12.5 \pm 3.2 \mathrm{~kg}$ versus $-9.2 \pm 2.0 \mathrm{~kg}, \mathrm{P}<0.001)$ and relative $(-10.2 \pm 2.0 \%$ versus $-8.3 \pm 1.6 \%, \quad \mathrm{P}<0.01)$ weight loss than the women (Table 1 ). The men also presented a higher absolute variation for whole-body LTM and trunk FM and a lower absolute variation for whole-body and trunk LTM/FM compared with the women.

For the whole population, absolute and relative variations in area, BMC and aBMD between baseline and 1 month after SG and according to anatomical site are presented in Table 2 and Fig. 1. An increase in both absolute and relative variations was observed for area, BMC and BMD at the lumbar spine and for area and $\mathrm{BMC}$ at the whole body, whereas a decrease for aBMD was observed at the hip. No variation was reported for the radius

As the body composition change differed between the men and women, specifically in the trunk region, which may potentially have modified the aBMD measurements, a subgroup analysis was performed according to sex (Table 3). The men and women showed similar profiles of variation, except for BMC at the whole body and lumbar spine, with the men presenting a significantly higher percentage of relative variation than the women and a tendency for lumbar spine aBMD $(P=0.057)$. Moreover, the radius area decreased significantly only in men.

Correlations between the absolute variations in anthropometric parameters (weight, BMI, LTM, FM) at various sites and the absolute variations in aBMD at the lumbar spine and total hip are presented in Table 4 and Fig. 1. Briefly, the absolute variations in $\mathrm{aBMD}$ at the lumbar spine were negatively correlated with the variations in weight, whole-body FM and trunk FM and positively with variations in whole-body LTM/
FM. However, when a multiple linear regression analysis was performed, only trunk FM remained an independent parameter associated with the variation in $\mathrm{aBMD}$ at the lumbar spine $(P=0.0109)$. The absolute variation in $\mathrm{aBMD}$ at the total hip was not correlated with the variations in any parameter. When the correlations were analysed according to sex (Table 5), the absolute variation in aBMD at the lumbar spine was negatively correlated with the variations in weight, BMI, whole-body FM and trunk FM in the women. In both sexes, the variation in $\mathrm{BMD}$ at the hip was correlated with the variation in lower limb LTM/FM, positively in women and negatively in men.

\section{Discussion}

In this study, we report that the great reduction in body weight and soft tissue in the first month following SG is associated with a modest variation in aBMD measurements that may have been influenced by anatomical site and sex. However, these variations in aBMD observed at the lumbar spine and total hip were close to the LSC, thus suggesting minimal clinical importance.

In the first months following SG, we observed substantial body weight loss of around $10 \mathrm{~kg}$, corresponding to a reduction of $-8.7 \%$ in the initial body weight and $-11 \mathrm{~cm}$ in the waist circumference (Maimoun et al., 2017). The soft tissue loss was composed of approximately equal proportions of FM and LTM, resulting in a lack of variation in FM/LTM (Maimoun et al., 2017). Although theoretically not sufficient to impact bone tissue, as demonstrated by the limited variation in bone remodelling markers in such a short period (Muschitz et al., 2015), the substantial body composition change 1 month after SG offers a unique opportunity to evaluate the effect of soft tissue on aBMD measurements measured with DXA in the clinical situation of obesity.

We observed that aBMD increased modestly but significantly at the lumbar spine, decreased slightly at the total hip and did not vary at the whole body or radius when the entire population was studied. Our multi-site evaluation represents progress, as earlier studies principally focused their analyses only on the lumbar spine (Svendsen et al., 2002) or femur (Binkley et al., 2003), and therefore could not account for the probable localized effects of soft tissue variation on aBMD measurements. It was recommended that sites other than the lumbar spine, such as peripheral sites, should be considered when examining the impact of weight loss on aBMD (Yu et al., 2012). In our study, we observed that the lumbar spine was most impacted by the loss of soft tissue, presenting a higher relative variation in BMD of $+2.45 \%\left(+0.02 \mathrm{~g} \mathrm{~cm}^{-2}\right)$ compared with the total hip $\left(-1.47 \% ;-0.02 \mathrm{~g} \mathrm{~cm}^{-2}\right)$, for an in vivo accuracy error of DXA that is comparable between bone sites (Svendsen et al., 1995). Nevertheless, we note that, despite being significant, these aBMD variations were close to the LSC, suggesting low clinical impact. The longitudinal variation in $\mathrm{aBMD}$ at the lumbar spine following intervention is the most subject to debate. Depending on the study, spine 

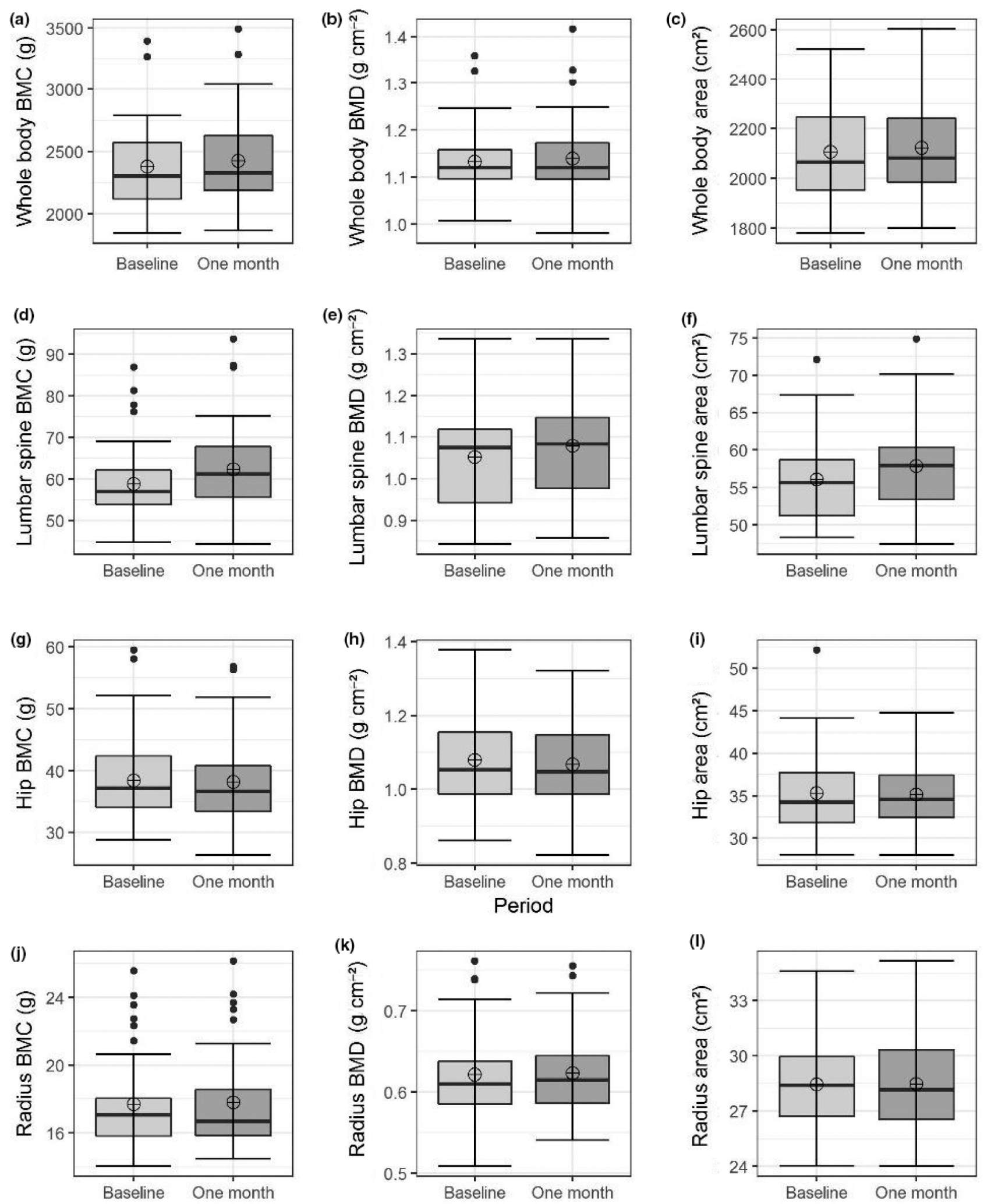

Figure 1 Area, bone mineral content and areal bone mineral density of the patients at baseline and 1 month after sleeve gastrectomy. Data are presented as aBMD, bone mineral density; BMC, bone mineral content. Lower whisker represents the smallest observation greater than or equal to lower hinge-1.5* IQR. Upper whisker represents the largest observation less than or equal to upper hinge $+1.5 *$ IQR. Lower and upper hinges represent, respectively, the $25 \%$ and the $75 \%$ quartile values. The open circles represent the mean value in each group.Data beyond the end of the whiskers are called outliers points and are plotted individually. IQR, interquartile range. 

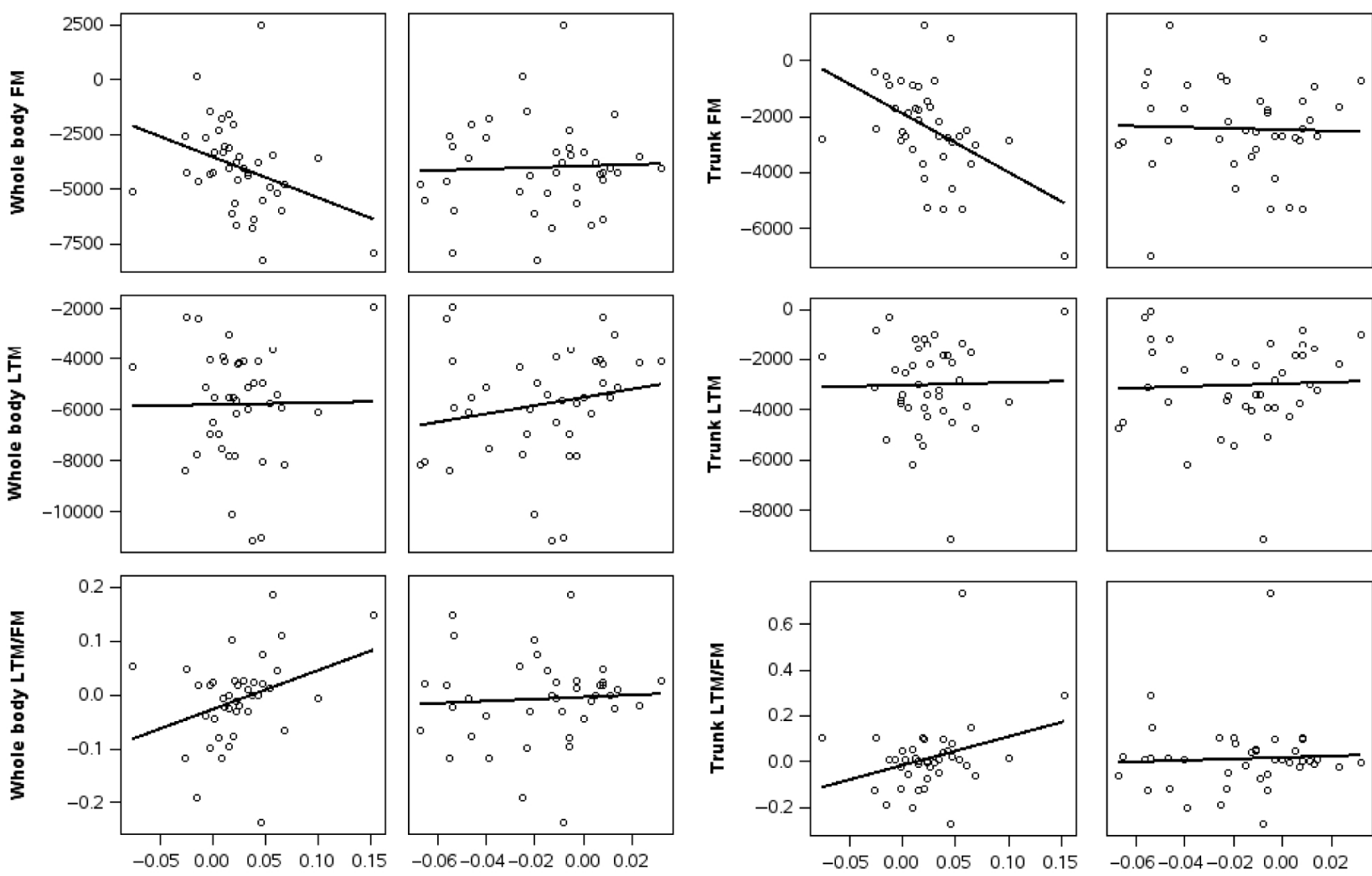

BMD L1-L4

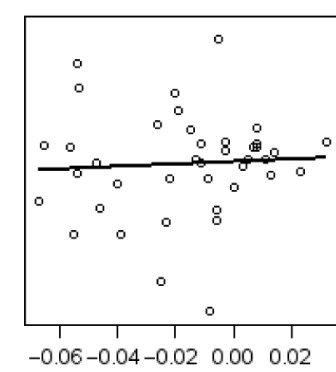

BMD hip
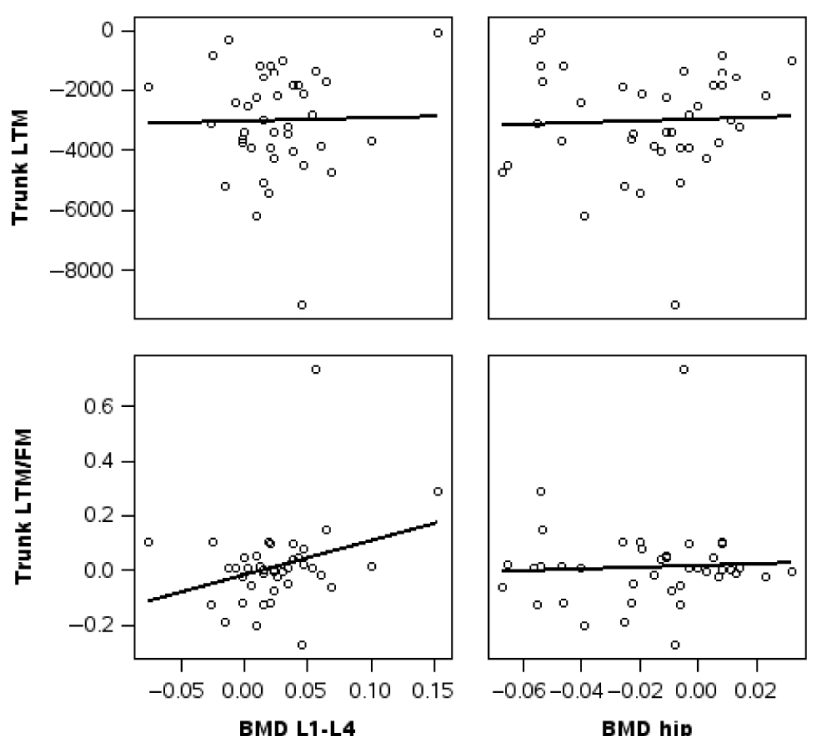

BMD hip

Figure 2 Correlation between the absolute changes in body composition and the absolute aBMD changes at lumbar spine and hip. BMD, bone mineral density; FM, fat mass; L1-L4, lumbar spine; LTM, lean tissue mass.

aBMD has been found to decrease (Svendsen et al., 1993, 1995, 2002) or increase (Pugnale et al., 2003; Ruiz-Tovar et al., 2013; Adamczyk et al., 2015). These divergent results call attention to an unresolved issue: although artefacts are known to be associated with DXA-based aBMD measurements in obesity and during weight loss (Bolotin, 1998, 2001, 2004, 2007; Javed et al., 2008, 2009; Knapp et al., 2012; Yu et al., 2012), it is not clear whether soft tissue induces an overestimation or underestimation of aBMD measurement. However, in line with our results, experimental studies have reported that the addition of $7.5 \mathrm{~kg}$ of fat layering in normal-weight subjects induced a decrease of $-2.2 \pm 3.7 \%$ aBMD at the lumbar spine but did not alter aBMD at the total hip or femoral neck (Yu et al., 2012). Using the same approach, another study demonstrated that the addition of localized 1-kg lard packets resulted in an aBMD decrease at the lumbar spine $(-1.9 \%)$ and a minimal change at the proximal femur $(+0.7 \%)$ (Evans et al., 2006). Moreover, it was likely that the quantity rather than the type (solid versus liquid) of fat influenced the aBMD measurements. Also, the addition of low exogenous soft tissue (i.e. $900 \mathrm{~g}$ lard or $1.2 \mathrm{l}$ oil) in normal-weight subjects had a limited but non-significant effect on aBMD values, whereas the placement of exogenous water $(1.4 \mathrm{l})$ to mimic extracellular fluid induced a significant decrease in lumbar spine BMD (Kim et al., 2012). These results suggested that the type of body fluid changes affected the aBMD measurements differently (Kim et al., 2012). Other experimental studies using different types of phantom showed that the surrounding placement of various liquids to mimic the influence of different soft tissues also induced an underestimation of aBMD (Javed et al., 2008, 2009; Kim et al., 2012). Our results suggest that aBMD measurements at the lumbar spine are more affected by FM than LTM, and the relative variation in trunk FM appeared as the only independent variable associated with the relative variation in aBMD at this skeletal site. Similarly, Cherif et al. (2018) recently compared in vivo and ex vivo rat lumbar spine and demonstrated that aBMD was underestimated by $8.74 \%$ when it was measured in vivo, and the difference between the two conditions was accentuated in overweight rats. This suggested, as in our study, that obesity induces an underestimation of aBMD and that abdominal fat may be an independent factor of the error in DXA aBMD measurements. Moreover, an interesting point that is observed in this study is that the aBMD changes at the lumbar spine were very heterogeneous $( \pm 3.44 \%)$. Thus, the accuracy errors in spine aBMD measurements may be caused by the non-homogenous distribution of adipose tissue in the abdomen, which is highly individual and differs from patient to patient.

However, conversely to our results, fat mass accumulation induced an overestimation of aBMD in other clinical or 


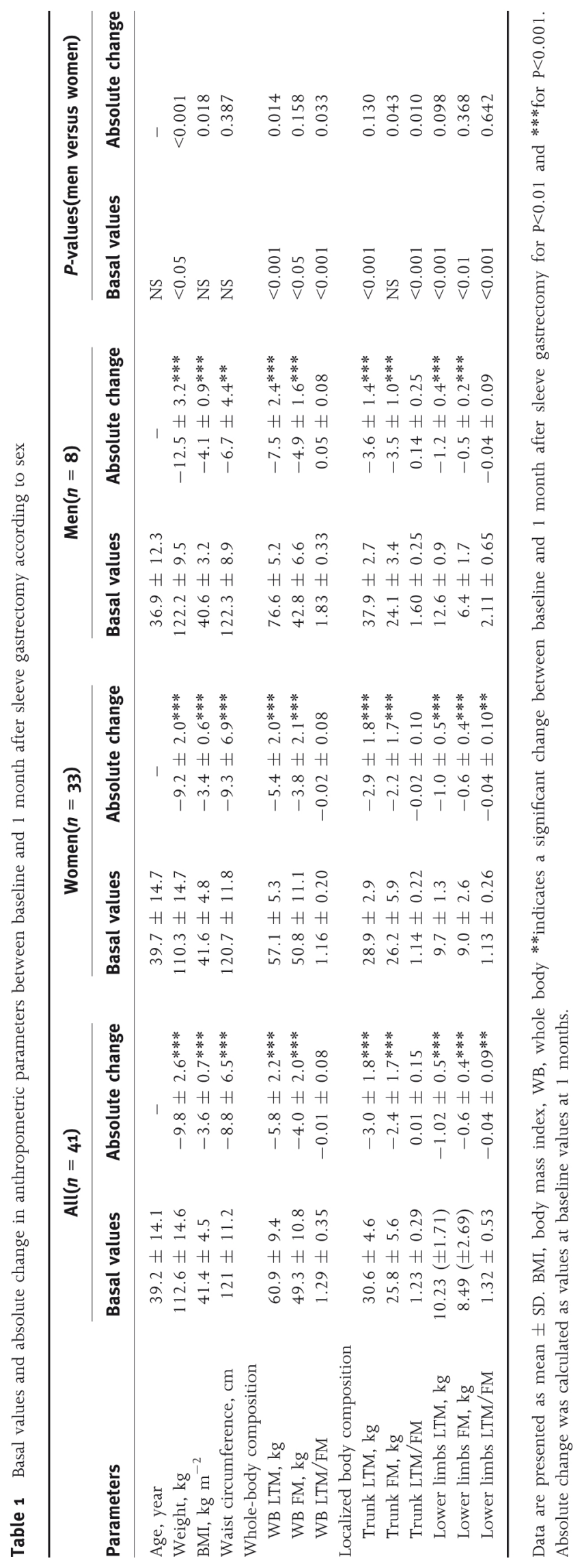


Table 2 Area, bone mineral content and areal bone mineral density of the patients at baseline and 1 month after sleeve gastrectomy

\begin{tabular}{|c|c|c|c|c|c|c|}
\hline & \multicolumn{6}{|c|}{ All patients $(n=40)$} \\
\hline & Baseline & 1 month & $\begin{array}{l}\text { Variation } \\
\text { (1 month -initial) }\end{array}$ & $\begin{array}{l}P \text {-value of } \\
\text { change }\end{array}$ & $\begin{array}{l}\% \text { of relative } \\
\text { change }^{a}\end{array}$ & $\begin{array}{l}P \text {-value of } \\
\text { relative change }\end{array}$ \\
\hline \multicolumn{7}{|l|}{ Whole body } \\
\hline Area, $\mathrm{cm}^{2}$ & $2106.2 \pm 187.6$ & $2122.0 \pm 182.2$ & $18.8 \pm 43.3$ & 0.010 & $0.94 \pm 2.09$ & 0.008 \\
\hline BMC, g & $2378.5 \pm 327.0$ & $2423.6 \pm 344.0$ & $35.4 \pm 62.2$ & $<0.001$ & $1.45 \pm 2.49$ & $<0.001$ \\
\hline $\mathrm{BMD}, \mathrm{g} \mathrm{cm}^{-2}$ & $1.13 \pm 0.07$ & $1.14 \pm 0.08$ & $0.00 \pm 0.03$ & 0.397 & $0.29 \pm 2.43$ & 0.451 \\
\hline \multicolumn{7}{|l|}{ Lumbar spine } \\
\hline Area, $\mathrm{cm}^{2}$ & $56.0 \pm 5.4$ & $57.9 \pm 6.1$ & $1.8 \pm 2.0$ & $<0.001$ & $3.25 \pm 3.44$ & $<0.001$ \\
\hline $\mathrm{BMC}, \mathrm{g}$ & $58.8 \pm 9.3$ & $62.3 \pm 11.0$ & $3.5 \pm 3.3$ & $<0.001$ & $5.70 \pm 5.29$ & $<0.001$ \\
\hline $\mathrm{BMD}, \mathrm{g} \mathrm{cm}^{-2}$ & $1.05 \pm 0.11$ & $1.08 \pm 0.11$ & $0.02 \pm 0.04$ & $<0.001$ & $2.45 \pm 3.44$ & $<0.001$ \\
\hline \multicolumn{7}{|l|}{ Hip } \\
\hline Area, $\mathrm{cm}^{2}$ & $35.3 \pm 5.9$ & $35.1 \pm 4.2$ & $0.2 \pm 1.5$ & 0.423 & $0.66 \pm 4.56$ & 0.400 \\
\hline $\mathrm{BMC}, \mathrm{g}$ & $38.3 \pm 7.4$ & $38.1 \pm 7.0$ & $-0.42 \pm 1.8$ & 0.150 & $-0.87 \pm 4.55$ & 0.235 \\
\hline $\mathrm{BMD}, \mathrm{g} \mathrm{cm}^{-2}$ & $1.08 \pm 0.13$ & $1.07 \pm 0.12$ & $-0.02 \pm 0.03$ & $<0.001$ & $-1.47 \pm 2.28$ & $<0.001$ \\
\hline \multicolumn{7}{|l|}{ Radius } \\
\hline Area, $\mathrm{cm}^{2}$ & $28.4 \pm 2.6$ & $28.5 \pm 2.7$ & $-0.02 \pm 0.85$ & 0.898 & $-0.02 \pm 2.98$ & 0.964 \\
\hline BMC, $g$ & $17.7 \pm 2.8$ & $17.8 \pm 2.9$ & $0.03 \pm 0.4$ & 0.591 & $0.15 \pm 2.33$ & 0.684 \\
\hline $\mathrm{BMD}, \mathrm{g} \mathrm{cm}^{-2}$ & $0.62 \pm 0.06$ & $0.62 \pm 0.05$ & $0.00 \pm 0.01$ & 0.531 & $-0.18 \pm 2.08$ & 0.595 \\
\hline
\end{tabular}

Data are presented as mean $\pm \mathrm{SD}$.

$\mathrm{a}_{\%} \%$ of relative change is defined as $100 *(1$ month - presurgery $) /$ presurgery $\left.)\right]$.

experimental conditions (Weigert \& Cann, 1999; Javed et al., 2009; Yu et al., 2012). For example, Yu et al. (2012) demonstrated with a spine phantom that DXA aBMD increased linearly with sequential fat layering. Similarly, a study in ex vivo defatted bone showed that the measurement of BMD was increased when it was surrounded by lard layers (Javed et al., 2009). Moreover, the close linear relationship between measured $\mathrm{aBMD}$ and the quantity of fat surrounding bone suggested that the absorption of photons by fat gives a spuriously high reading of aBMD (Javed et al., 2009). However, these studies explored only the effect of one component (i.e. FM) (Evans et al., 2006; Yu et al., 2012), and it is probable that layers of lard do not reproduce the physiological state of the patient's body, which is constituted of fat, muscle and organs, particularly at the abdomen (Cherif et al., 2018).

In our study, we evaluated LTM and FM simultaneously and, although the two components decreased concomitantly, only the FM variation was negatively associated with the aBMD variation at the lumbar spine and whole body. More precisely, the variation in $\mathrm{aBMD}$ at the lumbar spine was preferentially affected by a localized variation in FM at the trunk rather than at another site. This finding reinforces the observation that the DXA error of lumbar aBMD underestimation in obese patients is directly related to body weight, and more specifically to trunk FM. An animal study using different types of nutritional approach also reported that the difference between ex vivo and in vivo DXA lumbar measurement was due to weight, and specifically abdominal FM (Cherif et al., 2018). Our study, which evaluated men and women with a specific distribution pattern of accreted or lost fat mass (Ley et al., 1992; Maimoun et al., 2017), particularly at the ventral abdominal region (Maimoun et al., 2017), helps to specify the role of FM in aBMD measurement. The men of our study showed both a greater decrease in FM at the trunk and a greater increase in BMC and BMD at the lumbar spine compared with the women, a finding that reinforces our hypothesis of the potential effects of FM on aBMD measurements. Nevertheless, no correlation between the FM variation at the trunk and the $\mathrm{aBMD}$ variation at the lumbar spine was observed in the men, probably due to a lack of statistical power, whereas this correlation persisted in the women.

Other factors have been suggested to explain the inaccuracy of aBMD measurements in obese patients. These include the non-uniformities of the FM/LTM ratio that may be modified after, for example the addition of external fat mass ( $Y u$ et al., 2012). This technical limitation is due to DXA's use of two photon energies and, although DXA provides three body composition measurements (FM, LTM and BMC), it does not directly and simultaneously measure all three components. It was estimated that in a whole-body scan, $40 \%-45 \%$ of the pixels contain bone, thus distinguishing bone only from soft tissue (FM + LTM). The remaining pixels, containing the pure soft tissue that is adjacent to bone, are used to calculate FM/ LM (Shepherd et al., 2012; Bazzocchi et al., 2016), which may be modified by obesity and thereby induce an alteration in aBMD measurements (Bolotin, 1998, 2001, 2004, 2007; Yu et al., 2012). This explanation does not seem to apply to our context because no LTM/FM variation, particularly at the trunk, was observed after surgery when the whole population was studied. More probably, the high proportion of soft tissue may have led to an error in the measurement of the ROI. Also, we reported a concomitant increase in area and aBMD in 


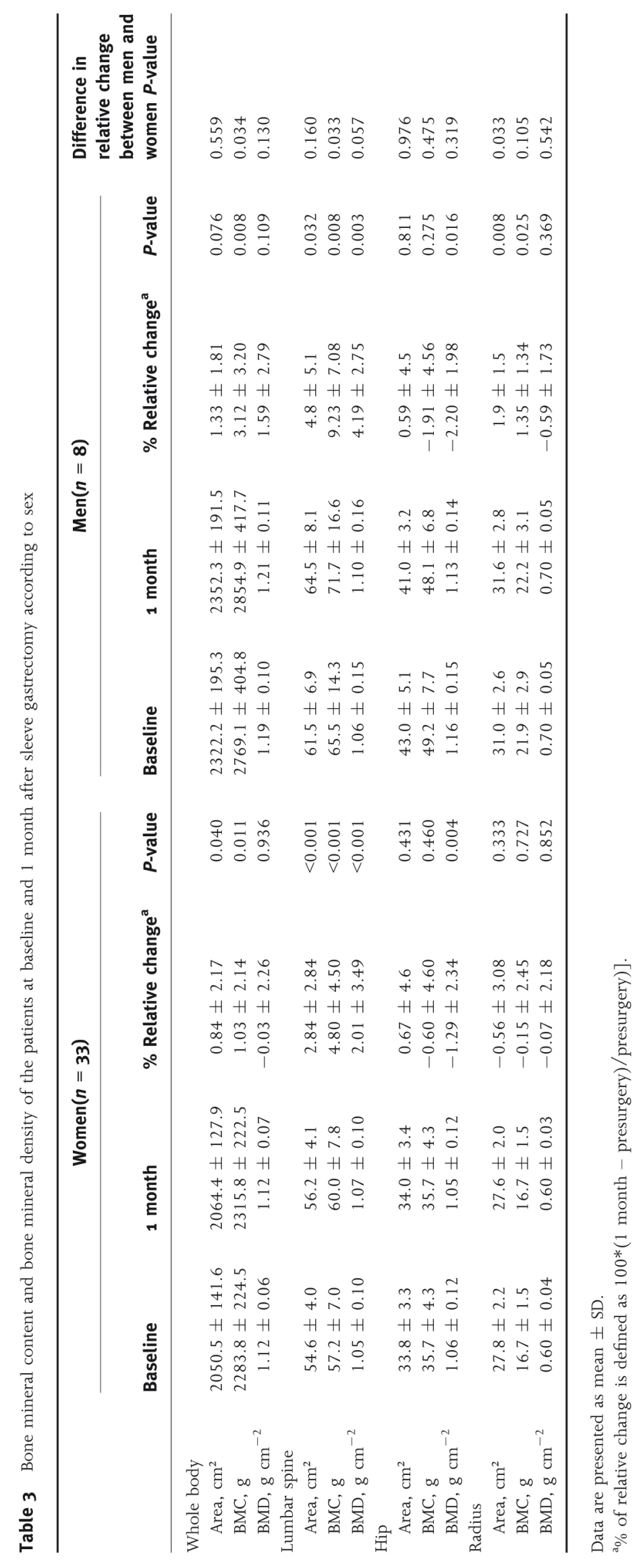


Table 4 Correlation analysis of the absolute changes in anthropometric parameters and the absolute areal bone mineral density changes at lumbar spine and hip for the whole population

\begin{tabular}{lcc}
\hline Variables & $\Delta$ BMD lumbar spine & $\Delta$ BMD hip \\
\hline$\Delta$ Weight & $-0.44^{* *}$ & 0.21 \\
$\Delta$ BMI & -0.30 & 0.16 \\
$\Delta$ Waist circumference, cm & 0.28 & 0.13 \\
$\Delta$ Whole-body FM, g & $-0.45^{* *}$ & 0.05 \\
$\Delta$ Whole-body LTM, g & -0.05 & 0.19 \\
$\Delta$ Whole-body LTM/FM & $0.34^{*}$ & 0.06 \\
$\Delta$ Trunk FM, g & $-0.48^{* *}$ & -0.03 \\
$\Delta$ Trunk LTM, g & -0.01 & 0.04 \\
$\Delta$ Trunk LTM/FM & 0.23 & 0.00 \\
$\Delta$ Lower limbs FM, g & 0.06 & -0.02 \\
$\Delta$ Lower limbs LTM, g & -0.07 & 0.18 \\
$\Delta$ Lower limbs LTM/FM & 0.02 & 0.15 \\
\hline
\end{tabular}

Data are presented as Pearson's or Spearman's correlation coefficients. FM, fat mass; LTM, lean tissue mass. *indicates a significant correlation between variations at $\mathrm{P}<0.05,{ }^{* *}$ for $\mathrm{P}<0.01$. BMI, body mass index.

Table 5 Correlation analysis of the absolute changes in anthropometric parameters and the absolute areal bone mineral density changes at lumbar spine and hip according to sex

\begin{tabular}{|c|c|c|c|c|}
\hline \multirow[b]{2}{*}{ Variables } & \multicolumn{2}{|c|}{ Women $(n=33)$} & \multicolumn{2}{|c|}{$\operatorname{Men}(n=8)$} \\
\hline & $\begin{array}{l}\triangle \text { BMD } \\
\text { lumbar } \\
\text { spine }\end{array}$ & $\begin{array}{l}\triangle \text { BMD } \\
\text { hip }\end{array}$ & $\begin{array}{l}\triangle \text { BMD } \\
\text { lumbar } \\
\text { spine }\end{array}$ & $\begin{array}{l}\triangle \text { BMD } \\
\text { hip }\end{array}$ \\
\hline$\Delta$ Weight & $-0.42 *$ & -0.03 & 0.26 & 0.29 \\
\hline$\Delta \mathrm{BMI}$ & $-0.38^{*}$ & -0.04 & 0.52 & -0.10 \\
\hline$\Delta$ Waist circumference, $\mathrm{cm}$ & 0.26 & 0.21 & 0.29 & 0.06 \\
\hline$\Delta$ Whole-body FM, g & $-0.43^{*}$ & 0.21 & 0.05 & 0.21 \\
\hline$\Delta$ Whole-body LTM, g & -0.02 & -0.07 & 0.55 & 0.10 \\
\hline$\Delta$ Whole-body LTM/FM & 0.22 & 0.00 & 0.26 & 0.07 \\
\hline$\Delta$ Trunk FM, g & $-0.35^{*}$ & -0.30 & -0.17 & -0.40 \\
\hline$\Delta$ Trunk LTM, g & -0.02 & 0.09 & 0.57 & 0.21 \\
\hline$\Delta$ Trunk LTM/FM & 0.03 & 0.31 & 0.24 & 0.17 \\
\hline$\Delta$ Lower limbs FM, g & 0.04 & 0.23 & 0.02 & 0.33 \\
\hline$\Delta$ Lower limbs LTM, g & -0.04 & 0.26 & 0.24 & -0.64 \\
\hline$\Delta$ Lower limbs LTM/FM & 0.01 & $0.39 *$ & 0.26 & $-0.81^{*}$ \\
\hline
\end{tabular}

Data are presented as Pearson's or Spearman's correlation coefficients. FM, fat mass; LTM, lean tissue mass. *indicates a significant correlation between variations of aBMD at lumbar spine and hip and various variables for $\mathrm{P}<0.05$. BMI, body mass index.

the same proportion at the lumbar spine, but not at the hip. Similar results were previously reported in an experimental study (Cherif et al., 2018). Other factors, which could not be evaluated in this study, have also been advanced to explain the error in BMD measurements. These may be due an inhomogeneous distribution of soft tissue throughout the scan ROI (Bolotin, 1998, 2001, 2004, 2007) and changes in marrow composition (Bolotin, 1998, 2001, 2004, 2007) associated with extra-osseous fat/lean tissue areal density ratio (Bolotin, 1998, 2001, 2004, 2007). Increased marrow adipose tissue has been found in obesity (Bredella et al., 2011, 2013, 2017) and 12 months after SG (Bredella et al., $2011,2013,2017)$. It is interesting to note that in Bredella et al.'s study (Bredella et al., 2017), marrow adipose tissue increased only at lumbar spine L1-L2, but not at the femoral metaphyseal region, a bone site close to the total hip where aBMD was measured in our study. However, the authors (Bredella et al., 2017) suggested that the lack of change in marrow adipose tissue after Roux-en-Y gastric bypass (RYGB) indicated that there was no systematic artefact from the marrow adipose tissue alterations on the DXA aBMD measurements, even though the two bariatric surgeries (i.e. RYGB and SG) induce a similar reduction in aBMD. Nevertheless, relatively small changes in marrow composition can have a marked effect on X-ray absorptivity (Bolotin, 1998, 2001, 2004, 2007), and limited and divergent results (Schafer et al., 2015; Bredella et al., 2017) on the effects of bariatric surgery on marrow adipose tissue have been reported. Therefore, other studies should be performed to clarify the effect of marrow on aBMD measurement.

Our study presented some limitations that should be noted. Notably, although the patients presented acute body weight loss, BMI, waist circumference and fat mass remained higher 1 month after SG than the values in a normal-weight population, indicating a persistent effect of soft tissue on the aBMD measurements. To explore this issue, a re-evaluation of these patients would be interesting after the loss of the total excessive weight. However, this might take 1-2 years, a time period during which a variation in bone mass, measured by both DXA and quantitative computed tomography (QCT), has been observed (Muschitz et al., 2015; Bredella et al., 2017). In addition, the imbalance in the numbers of patients of the two sexes did not allow precise discrimination of the role of specific fat mass localization on BMD. Moreover, the effect of FM variation on the lumbar spine aBMD measurements we obtained cannot be generalized to all DXA devices produced by other manufacturers (i.e. Nordland or Lunar) because other techniques for X-ray production were used. It is also probable that software versions and subsequent algorithms are more or less sensitive to the variation in FM.

In conclusion, this study shows some evidence of the effects of body composition, particularly FM, on aBMD. However, given the modest change in aBMD in relation to the precision error of aBMD measurements, it appears that significant weight loss does not have a clinically significant impact on evaluation of aBMD using DXA.

\section{Conflict of Interest}

Laurent Maïmoun, Denis Mariano-Goulart, Audrey Jaussent, Patrick Lefebvre, Marie-Christine Picot, Krishna Mahadea, Vincent Boudousq, Clémence Fouillade, David Nocca and Fayçal Ben Bouallègue declare that they have no conflict of interest. 


\section{References}

Adamczyk P, Buzga M, Holeczy P, et al. Bone mineral density and body composition after laparoscopic sleeve gastrectomy in men: a short-term longitudinal study. Int J Surg (2015); 23: 101-107.

Bazzocchi A, Ponti F, Albisinni U, et al. DXA: technical aspects and application. Eur J Radiol (2016); 85: 1481-1492.

Binkley N, Krueger D, Vallarta-Ast N. An overlying fat panniculus affects femur bone mass measurement. J Clin Densitom (2003); 6: 199-204.

Bolotin HH. A new perspective on the causal influence of soft tissue composition on DXA-measured in vivo bone mineral density. J Bone Miner Res (1998); 13: 1739 1746.

Bolotin HH. Inaccuracies inherent in dual-energy X-ray absorptiometry in vivo bone mineral densitometry may flaw osteopenic/ osteoporotic interpretations and mislead assessment of antiresorptive therapy effectiveness. Bone (2001); 28: 548-555.

Bolotin HH. The significant effects of bone structure on inherent patient specific DXA in vivo bone mineral density measurement inaccuracies. Med Phys (2004); 31: 774-788.

Bolotin HH. DXA in vivo BMD methodology : an erroneous and misleading research and clinical gauge of bone mineral status, bone fragility, and bone remodeling. Bone (2007); 41: 138-154.

Bredella MA, Torriani M, Ghomi RH, et al. Determinants of bone mineral density in obese premenopausal women. Bone (2011); 48: 748-754.

Bredella MA, Gill CM, Gerweck AV, et al. Ectopic and serum lipid levels are positively associated with bone marrow fat in obesity. Radiology (2013); 269: 534-541.

Bredella MA, Greenblatt LB, Eajazi A, et al. Effects of Roux-en-Y gastric bypass and sleeve gastrectomy on bone mineral density and marrow adipose tissue. Bone (2017); 95: 85-90.

Cherif R, Vico L, Laroche N, et al. Dual-energy X-ray absorptiometry underestimates in vivo lumbar spine bone mineral density in overweight rats. $J$ Bone Miner Metab (2018); 36: 31-39.

Evans EM, Mojtahedi MC, Kessinger RB, et al. Simulated change in body fatness affects Hologic QDR 4500A whole body and central DXA bone measures. J Clin Densitom (2006); 9: 315-322.

Hansen MA, Overgaard K, Christiansen C. Spontaneous postmenopausal bone loss in different skeletal areas-followed up for 15 years. J Bone Miner Res (1995); 10: 205210.

Javed F, Wang J, Colt E, et al. The validity of iDXA in determining bone mineral content (BMC), bone mineral density (BMD) and body fat (BF with changes in subcutaneous tissue thickness (STT). Int J Body Compos Res (2008); 6: 74.

Javed F, Yu W, Thornton J, et al. Effect of fat on bone measurement of bone mineral density. Int J Body Compos Res (2009); 7: 37-40.

Jensen MD, Ryan DH, Apovian CM, et al. 2013 AHA/ACC/TOS guideline for the management of overweight and obesity in adults: a report of the American College of Cardiology/American Heart Association Task Force on Practice Guidelines and The Obesity Society. J Am Coll Cardiol (2013); 63: 2985-3023.

Kim KN, Kim BT, Kim KM, et al. The influence of exogenous fat and water on lumbar spine bone mineral density in healthy volunteers. Yonsei Med J (2012); 53: 289293.

Knapp KM, Welsman JR, Hopkins SJ, et al. Obesity increases precision error in dual-energy X-ray absorptimetry measurements. J Clin Densitom (2012); 15: 315-319.

Ley CJ, Lees B, Stevenson JC. Sex- and menopause-associated changes in body-fat distribution. Am J Clin Nutr (1992); 55: 950-954.

Maimoun L, Mura T, Leprieur E, et al. Impact of obesity on bone mass throughout adult life: influence of gender and severity of obesity. Bone (2016); 90: 23-30.

Maimoun L, Lefebvre $\mathrm{P}$, Jaussent $\mathrm{A}$, et al. Body composition changes in the first month after sleeve gastrectomy based on gender and anatomic site. Surg Obes Relat Dis (2017); 13: 780-787.

Muschitz C, Kocijan R, MArterer C, et al. Sclerostin levels and changes in bone metabolism after bariatric surgery. J Clin Endocrinol Metab (2015); 100: 891-901

Picaud JC, Nyamugabo K, Braillon $\mathrm{P}$, et al. Dual-energy X-ray absorptiometry in small subjects: influence of dual-energy X-ray equipment on assessment of mineralization and body composition in newborn piglets Pediatr Res (1999); 46: 772-777.

Pugnale N, Giusti V, Suter $M$, et al. Bone metabolism and risk of secondary hyperparathyroidism 12 months after gastric banding in obese pre-menopausal women Int J Obes Relat Metab Disord (2003); 27: 110 116.

Ruiz-Tovar J, Oller I, Priego A, et al. Shortand mid-term changes in bone mineral density after laparoscopic sleeve gastrectomy. Obes Surg (2013); 23: 861-866.

Schafer AL, Li X, Schwartz AV, et al. Changes in vertebral bone marrow fat and bone mass after gastric bypass surgery: a pilot study. Bone (2015); 74: 140-145.

Shepherd JA, Fan B, Lu Y, et al. A multinational study todevelop universal standardization of whole-body bone density and composition using GE healthcare lunar and hologic DXA systems. J Bone Miner Res (2012); 27: 2012.

Soltani S, Hunter GR, Kazemi A, et al. The effects of weight loss approaches on bone mineral density in adults: a systemic review and meta-analysis of randomized controlled trials. Osteoporos Int (2016); 27: 2655-2671.

Svendsen OL, Hassager C, Christiansen C. Effect of an energy-restrictive diet, with or without exercise, on lean tissue mass, resting metabolic rate, cardiovascular risk factors, and bone in overweight postmenopausal women. Am J Med (1993); 95: $131-140$.

Svendsen OL, Hassager C, Skodt V, et al. Impact of soft tissue on in vivo accuracy of bone mineral measurements in the spine, hip, and forearm: a human cadaver study. J Bone Miner Res (1995); 10: 868-873.

Svendsen OL, Hendel HW, Gotfredsen A, et al. Are soft tissue composition of bone and non-bone pixels in spinal bone mineral measurements by DXA similar? Impact of weight loss. Clin Physiol Funct Imaging (2002); 22: 72-77.

Weigert JM, Cann CE. Dual energy X-ray absorptiometry (DXA) in obese patient: are normal values really normal? J Women Imaging (1999); 1: 11-17.

Yu EW, Thomas BJ, Brown JK, et al. Simulated increases in body fat and errors in bone mineral density measurements by DXA and QCT. J Bone Miner Res (2012); 27: 119-124. 